\title{
Relationship between Maturity and Isoenzymes of Erythrocytic Carbonic Anhydrase in Newborn Infants
}

\author{
J. B. MOYNIHAN"11ir \\ Department of Physiolegg. University College Dublin, Dublin, Ireland
}

\begin{abstract}
Summary
The present study was carried out to assess the activities of all three principal isoenzymes of human erythrocytic carbonic anhydrase (HCA) in newborn infants in relation to their estimated gestational ages. Blood samples were collected at parturition from the umbilical cords of $\mathbf{4 5}$ normal healthy infants. Among the samples taken before day 290 of gestation, the activity ratio (isoenzyme B: isoenzyme $\dot{C}$ ) was correlated significantly with estimated gestational age. After day 290 , the range of $B: C$ activity ratios was similar to that observed in adults. Isoenzyme $\Lambda$ represented a relatively constant proportion (mean \pm SENI) at $7.2 \pm 0.2 \%$ of total $\mathrm{HC} \mathrm{C}$ activity. The $\mathrm{B}: \mathrm{C}$ activity ratio appears to be a more reliable and sensitive index of maturity than either the total HCA activity or the ratio of total HCA activity to the total hemoglobin concentration in cord blood.
\end{abstract}

\section{Speculation}

The progressive change in the ratio of activities of $\mathrm{HC} A$ isoenzymes 13 and $C$ in cord blood from day 253 to day 290 of gestation is of potential utility in the accurate estimation of gestational age within the range.

Human erythrocytic carbonic anhydrase catalyzes the reversible reaction

$$
\mathrm{H}_{2 .} \mathrm{O}+\mathrm{CO}_{2 .}=\mathrm{H}_{2.2} \mathrm{CO}_{3}
$$

and thereby accelerates the hydration of metabolic carbon dioxide in the capillaries of the tissues as well als the dehydration of carbonic acid in the capillaries of the lungs. Carbonic anhydrase activity is generally believed to be absent from plasma, but if the erythrocytic enzyme is totally inhibited, respiratory exchange of carbon dioxide is impaired and the levels of carbon dioxide in both tissues and capillary blood are elevaled (4). Logan et al. (3) Pound lower total levels of HCA activity in newborn infants compared with adults, and showed that total HCA activity tended to be lower in premature than in full term infants. Isoenzymes $A$ and $B$ of $\mathrm{HCA}$ exhibit immunologic identity. similar specific activities, and amino acid compositions, but differ in their electrophoretic mobilities. Isoenzyme C has molecular weight and zinc content similar to the other two principal isoenzymes, but has a greater specific activity and also differs in its detailed amino acid composition, electrophoretic mobility, and antigenic characteristies (4). In the blood of the newborn child. the properties of the three principal isoenzymes of HCA are similar to the properties of the adult isoenzymes (14). Wehinger (12) found that IICA activity was low in fetal blood until the 32 nd week of gestation, after which the synthesis of isoenzyme $C$ proceeded initially more rapidly than the synthesis of isoenzyme 13. There is probably a compensatory increase in isoenzyme 13 synthesis later, for the results of Norgaard-Pedersen and Klebe (6) show that the progressive increase in the isoenzyme ratio $\mathrm{B}: \mathrm{C}$ occurs between the 35 th and 39 th weeks. Those results were obtained in cord blood from the infants of diabetic mothers, but did not differ from results obtained by the same workers in newborn children of normal mothers. A recent investigation (5) failed to establish a useful precise relationship between the concentrations of the isoenzymes $B$ and $C$. as measured by an immunologic method. and the birth weight of the newborn child. The present study was carried out to assess the activities of all three principal isoenzymes of HCA in newborn infants in relation to their estimated gestational ages.

\section{MITHODS}

Blood samples were collected at parturition from the umbilical cords of 45 normal healthy infants, the gestational age of each child being estimated from the date of the mother's last menstrual period. In every case, the umbilical cord was sectioned between ligatures and the blood sample subsequently taken from the discarded placenta. Citrate was used as anticoagulant in the simples of whole blood, which were hemolyzed by repeated freczing and thawing and then stored at $-20^{\circ}$.

The three principal isoenzymes of HCA were separated from each hemolysate by vertical starch gel electrophoresis $(8 \mathrm{~V} / \mathrm{cm}$ for $16 \mathrm{hr}$ at $4^{\circ}$ ) using a discontinuous buffer system (7). After quantitative elution of the individual isoenzymes by maceration of the weighed starch gel segments in chilled isotonic saline solution, their activities were assayed (after appropriate dilution) at $\left(0^{\circ}\right.$ by the electrometric method of Wilbur and Anderson (13). The number of operational units (EU) of HCA activity present in the undiluted hemolysates was defined as

$$
E U=\frac{\left(R-R_{11}\right) \cdot \underline{F}}{R_{11}}
$$

where $R$ and $R_{11}$ were the rates of the assay reaction in the presence and the absence of the $11 C A$. respectively, and $F$ was the relevant dilution factor. Hemoglobin was determined in the hemolysates as cyanmethemoglobin (11). Regressions were computed by the method of least squares.

\section{RESULTS}

Data from make and female infants were combined, as no statistically significant differences between the sexes were found in the values determined in the investigation. The early samples of infants blood contained isoenzyme $B$ activity as a smaller proportion, and isoenzyme $C$ as a larger proportion of the total HCA activity compared with the blood from newborn infants of greater gestational age (Table 1). Isoenzyme A represented a relatively constant proportion (mean \pm SEM) at $7.2 \pm$ $0.2 \%$ of total IICA activity in all the samples taken in this study. Among the samples taken before day 290 of gestation, the activity ratio (isoenzyme 13 :isoenzyme $C$ ) was significantly correlated with estimated gestational age $(P<0.01, r=0.94)$ (Fig. 1). After daty 290 , the range of $B: C$ activity ratios, $0.93 \pm 0.01$ 
(mean \pm SEM $; 12$ observations), was similar to that found in adults, $0.97 \pm 0.02$ (mean \pm SEM; 45 observations).

Total HCA atctivity increased significantly with gestational age $(P<0.01, r=0.69)$ over the whole range investigated (Fig. 2),

Table 1. Activities of principal isoenzymes as percentages of total activity of erythrocytic carbonic anhydrase (HCA) in cord blood of newborns ${ }^{1}$

\begin{tabular}{ccccc}
\hline & No. of & \multicolumn{3}{c}{ Isoenzymes } \\
$\begin{array}{c}\text { Gestational } \\
\text { age, days }\end{array}$ & ples & A & B & C \\
\hline $251-260$ & 5 & $6.0 \pm 0.5$ & $32.6 \pm 0.9$ & $61.4 \pm 0.8$ \\
$261-270$ & 6 & $7.5 \pm 0.6$ & $36.6 \pm 1.4$ & $55.9 \pm 1.1$ \\
$271-280$ & 8 & $7.0 \pm 0.5$ & $41.0 \pm 0.9$ & $52.0 \pm 0.5$ \\
$281-290$ & 14 & $7.2 \pm 0.4$ & $43.8 \pm 0.3$ & $49.0 \pm 0.6$ \\
$291-301$ & 12 & $7.6 \pm 0.3$ & $44.2 \pm 0.4$ & $48.2 \pm 0.4$ \\
\hline
\end{tabular}

' Results are expressed as mean \pm SEM. and there was also a statistically significant correlation $(P<$ $0.01 . r=0.64)$ between the ratio of total HCA activity to hemoglobin concentration and gestational age (Fig. 3).

\section{DISCUSSION}

In the past, it has been thought that only isoenzymes $B$ and $C$ of HCA, may be found in newborn infants (9). However, isoenzyme $A$ is more difficult to separate and isolate and, because it exhibits immunologic identity with isoenzyme $B$, has been included in apparent measurements of the latter by immunologic methods. The techniques adopted in the present investigation demonstrated the presence of the three principal isoenzymes of HCA in all of the samples studied. Whereas the relative alctivity of isoenzyme $B$ tended to rise with increasing gestational age, isoenzyme $A$ represented a virtually constant proportion of total HCA activity in newborn infants, implying that the epigenetic modification of the $B$ isoenzyme to the $A$ form may not have

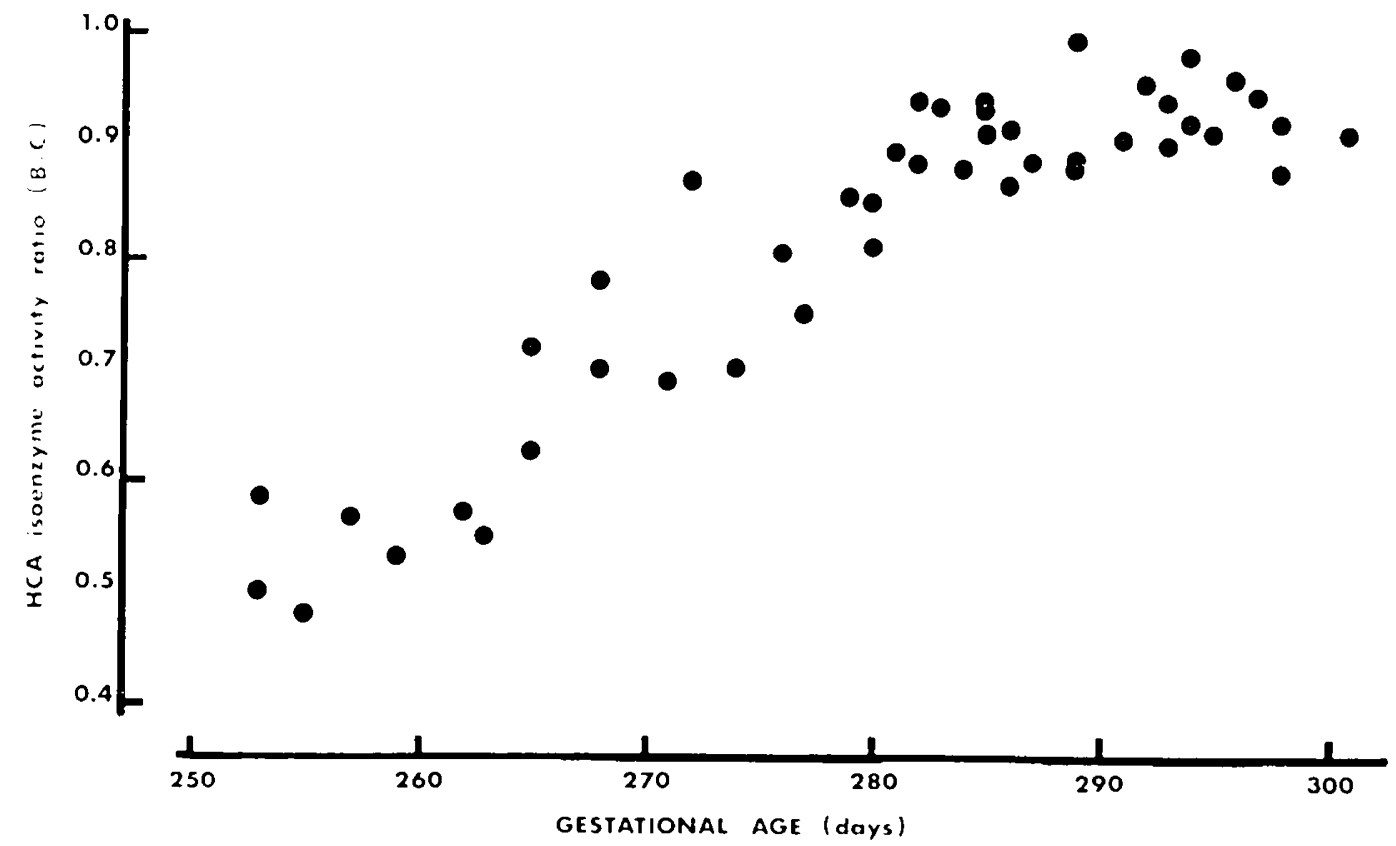

Fig. 1. Relationship between the ratio of activity of isoenzyme B to activity of isoenzyme $C$ of erythrocytic carbonic anhydrase (HCA) in cord blood of the newborn, and gestational age.

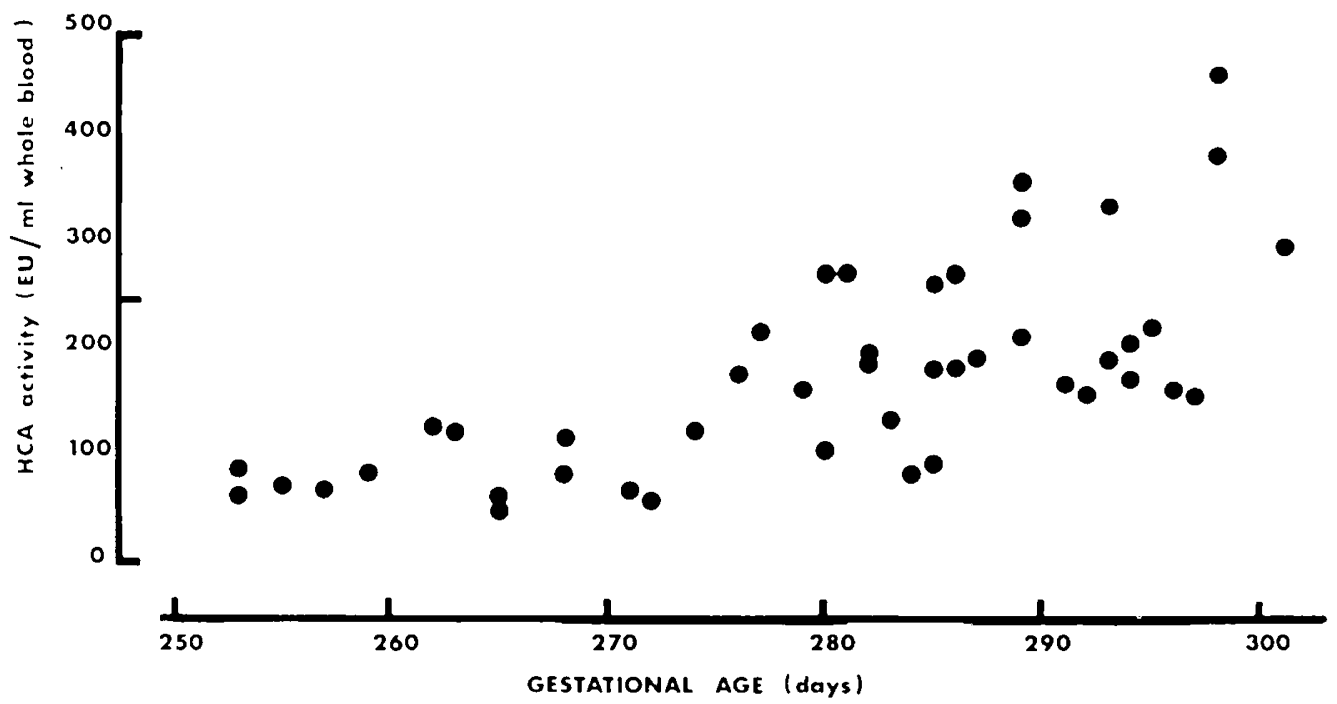

Fig. 2. Relationship between the total activity of erythrocytic carbonic anhydrase $(H C A)$ in cord blood of the newborn, and gestational age. EU: enzyme units. 


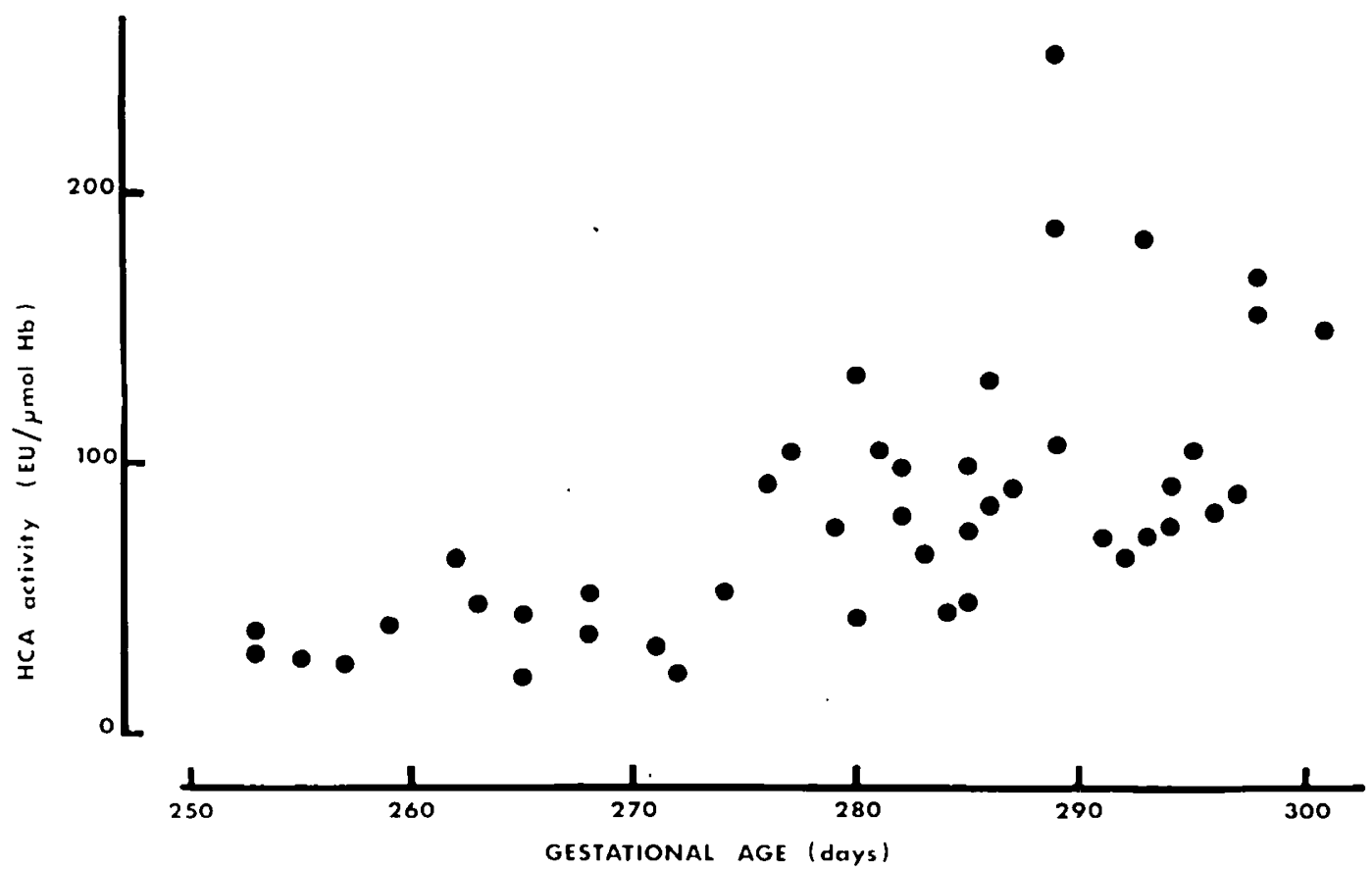

Fig. 3. Relationship between the ratio of total activity of erythrocytic carbonic anhydrase $(H C A)$ to the concentration of haemoglobin $(H b)$ in cord blood of the newborn, and gestational age. EU: enzyme units.

been entirely the random process observed in vitro by Funakoshi and Deutsch (2). Since the progressive increase in isoenzyme $B$ activity with advancing age during late gestation is inversely related to the activity of isoenzyme $C$, the $B: C$ activity ratio might be adopted as a measure of maturity of the newborn. This ratio appears to be a more reliable and sensitive index of maturity than either the total HCA activity $(10)$ or the ratio of total HCA activity to the total hemoglobin concentration in cord blood. A reliable indicator of gestational age has been sought in those situations in which pregnancy follows temporary oral contraceptive-induced amenorrhea (5) and those in which infants are born to diabetic women (who have a high incidence of irregular menstruation (6)), and in forensic applications. The techniques used in the present study appear to form the basis of such a test, for the electrometric method of assay of IICA activity is reasonably rapid. Combined with the use of starch gel electrophoresis to separate the individual isoenzymes of HCA, it has the advantage over immunologic methods of assay of enabling a more precise estimate of the $B: C$ ratio to be obtained, because isoenzyme $B$ may be determined specifically without interference due to the presence of isoenzyme $A$ in the assiay system. It is possible that the apparent reliability of this technique for predicting gestational age may be reduced in infants - with abnormal circulating levels of thyroid hormones, since in adults the levels of HCA isoenzyme $B$ are elevated in hypothyroid and reduced in hyperthyroid subjects (1). This phenomenon does not appear to occur in newborn infants, however, as the $B: C$ activity ratio in children with respiratory distress syndrome is normal (9) despite the impaired thyroid function evident in such infants (8). The potential clinical utility of estimations of the $\mathrm{B}: \mathrm{C}$ activity ratio in newborn infants is enhanced by the fact that the presence of diabetes (whether treated or not) in the mother does not alter the ratio in the child (6).

\section{CONCLUSION}

The ratio of activities of isoenzymes $B$ and $C$ of erythrocytic carbonic anhydrase in cord blood is significantly correlated with gestational age in the range of 253-290 days. This relationship may be useful in providing the basis of an index of maturity of the newborn.

\section{REFERENCES AND NOTES}

1. Anker, N., and Mondrup, M.: Carbonic anbydrase isoenzyme B in erythrocytes of subjects with thyroid disorders. Clin. Chim. Actil, 54: 277 (1974).

2. Funakoshi, S., and Deutsch, H. F.: Human carbonic anhydrases. II. Some physicochemical properties of native isozymes and of similar isozymes generated in vitro. J. Biol. Chem., 244: 3438 (1969)

3. Logan, R. W., Crooks, S. M., Hutchison, J. H., and Kerr, M. M.: Blood carbonic anhydrase activity in the newborn. Arch. Dis. Childhood, 48: 256 (1973).

4. Maren, T. H.: Carbonic anhydrase: chemistry, physiology, and inhibition. Physiol. Rev., 47: 595 (1967).

5. Mondrup. M., and Anker, N.: Carbonic anhydrase isoenzymes in the erythrocytes of new-born premature and full-term infants. Clin. Chim. Acta, 61: $\$ 27(1975)$.

6. Norgaard-Pedersen, B.. and Klebe, J. G.: Alpha-fetoprotein and carbonic anhydrase $B$ and $C$ concentration in cord blood from newhorn infants of diabetic mothers. Acta Endocrinol., 75: suppl. 182, 81 (1974).

7. Poulik, M. D.: Starch gel electrophoresis in a discontinuous system of buffers. Nature, 180: 1477 (1957).

8. Redding, R. A., and Pereira, C.: Thyroid function in respiratory distress syndrome of the newborn. Pediatries, 54: 423 (1974).

9. Sell, J. E., and Petering, H. G.: Carbonic anhydrases from human neonatal erythrocytes. J. Lab. Clin. Med., 84: 369 (1974).

10. Stevenson, S. S.: Carbonic anbydrase in newborn infants. J. Clin. Invest., 22: $403(1943)$.

11. van Kampen, E. J., and Zijlstra, W. G.: Standardization of hemoglobinometry. Il. The hemoglobincyanide method. Clin. Chim. Acta, 6: 538 (1961)

12. Wehinger, $\mathrm{H}$ : Zur natur und ontogenetischen entwicklung von carboanhydratse-iseculymen in menschlichen erythrosyten. Blut, 27: $172(197.3)$.

13. Wilbur, K. M., and Anderson, N. G.: Electrometric and colorimetric determination of carbonic anhydrase. J. Biol. Chem.. 176: 147 (1948).

14. Wistrand, P. J., and Rao, S. N.: Immunologic and kinetic properties of carbonic anhydrases from various tissues. Biochim. Biophys. Acta, 154: 130 (1968).

15. The assistance and cooperation of the Master, Dr. D. J. Meatgher, and the staff of the National Maternity Hospital, Dublin, are greatly appreciated.

16. Requests for reprints should be addressed to: J. B. Moynihan, Ph.D., Department of Physiology, University College Dublin, Earlsfort Terrace, Dublin (lreland).

17. Received for publication December 6, 1976

18. Accepted for publication January 19, 1977. 